oder Hinrichtung) werden deutlich. Der Meinungswandel vom Links- zum Rechtsliberalismus (z.B. Robert von Römer) oder umgekehrt (Johannes von Hieber), von Gegnerschaft eines Landesverbandes der Zentrumspartei zu deren Ehrenvorsitz (Rudolf Probst), vom führenden Oppositionellen zum Totengräber der Frankfurter Nationalversammlung (Friedrich von Römer) ist beeindruckend. Wie eng Publizistik und politische Aktivität verzahnt sind, lässt sich an der relativen Häufigkeit des ZweitBerufes Verleger (Otto Elben, Heinrich von Kraut, Theodor Körner) bzw. Journalist (Heinrich Kessler, Mathilde Planck, Friedrich Rödinger, Karl Friedrich Schnitzer, Kurt Schumacher, Eduard Süskind, Gottlob Tafel) ablesen. Neben Juristen sind Theologen gut vertreten, allerdings in breiter Streuung der politischen Lager (z.B. Christoph Blumhardt, Johannes von Hieber, Johann Baptist von Keller, Johannes Evangelist von Kuhn, Johann Baptist Sproll, Eduard Süskind, Theophil Wurm). Das veränderte Wahlrecht und die verstärkte Interessenvertretung ökonomisch definierter Bevölkerungsgruppen bringen im Hinblick auf die Sozialstruktur einen allmählichen Wandel vom Honoratiorenparlament zum Organ einer pluralen Massendemokratie mit sich.
Das Angebot wird abgerundet durch kurze Übersichtsseiten zu den Versammlungsorten und zu wichtiger Sekundärliteratur. Die inhaltliche Dokumentation wird zusammen mit den Digitalisaten der Landtagsprotokolle freigeschaltet.

\section{Digitale Präsentation}

Unterhalb der Einstiegsseite gelangt man per Link zu den einzelnen Bänden der Landtagsprotokolle. Jeder bibliographischen Einheit entspricht ein Goobi-Projekt, das mit einer verbalen Bezeichnung für die Teilserie, den Zeitraum und ggf. den Teilband eindeutig identifiziert wird. Sucht man z.B. nach dem Teilband 2 der Sitzungsprotokolle der Zweiten Kammer im Zeitraum 1907-1909, so ist dies in zweierlei Weise möglich. Primärer Sucheinstieg wird das betreffende Sitzungsjahr bzw. ein Zeitraum (z.B. die Legislaturperiode) sein. Mit dem Jahr sind alle bibliographischen Einheiten dieser zeitlichen Zuordnung unabhängig von der Teilserie verlinkt. Sekundär wird aber auch eine Liste aller Teilbände der jeweiligen Teilserie (z.B. Kammer 1 bzw. 2, jeweils Protokolle / Beilagen / Register) als Ausgangspunkt für die Recherche geboten.

Christian Herrmann

\title{
Die Kriegssammlung der Königlichen Hofbibliothek Stuttgart
}

\section{Die Anfänge der Kriegssammlung}

Im Ersten Weltkrieg entstanden zahlreiche sogenannte Kriegssammlungen, die zumeist versuchten, sämtliches Druckmaterial aus der "großen Zeit", die man angeblich miterleben durfte, zu sammeln. Diese Kriegssammlungen gab es sowohl an bereits bestehenden öffentlichen Bibliotheken, Archiven oder Museen als auch als Neugründungen von Privatleuten. Die bekannteste und größte Privatsammlung war wohl die sog. „Weltkriegsbücherei“ des schwäbischen Industriellen Richard Franck, die in Berlin gegründet wurde. Aus dieser Institution ging die heutige „Bibliothek für Zeitgeschichte" in Stuttgart hervor.
In Stuttgart befand sich zur damaligen Zeit neben der Königlichen Öffentlichen Bibliothek auch die Königliche Hofbibliothek. Ursprünglich als Handbibliothek von König Friedrich I. von Württemberg (1754-1816) zwischen 1806 und 1810 gegründet, war sie beim Tod Friedrichs schon auf 47.000 Bände angewachsen. ${ }^{1}$ Im Testament Friedrichs wurde die Bibliothek zum Staatseigentum erklärt mit Nutzungsrecht durch den württembergischen König. König Wilhelm I. von Württemberg bezeichnete sie 1816/17 als "Königliche Handbibliothek", daraus wurde 1887 die "Königliche Hofbibliothek". Die Bibliothek war in den Räumen der früheren Hohen Karlsschule untergebracht. 
Wie in vielen anderen Bibliotheken wurde nach 1914 auch in der Stuttgarter Hofbibliothek eine eigene "Kriegssammlung" angelegt. Prof. Karl Hayo von Stockmayer (1871-1948), der Leiter der Hofbibliothek, hatte schon mit Kriegsbeginn zu sammeln begonnen. In einem Schreiben an das Königliche Hofmarschallamt umriss Stockmayer im Juni 1916 auch, was er sammelte: „Ueber die Art der zu sammelnden Literatur lässt sich in Kürze sagen: Alles was auf den Weltkrieg Bezug hat."2

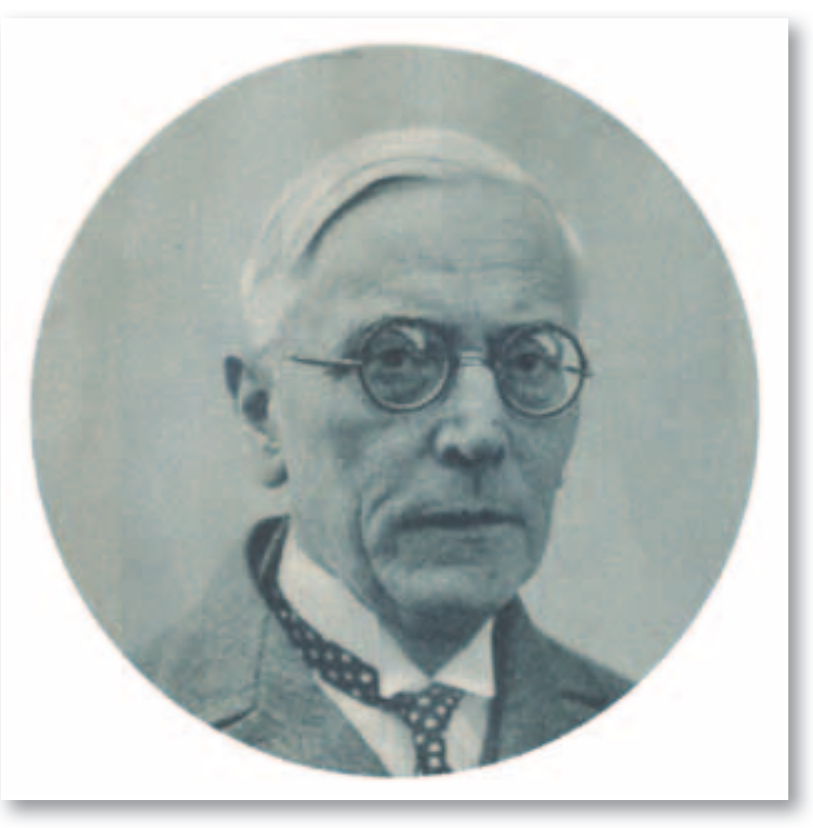

Prof. Karl Hayo von Stockmayer, der Leiter der Königlichen Hofbibliothek

Stockmayer war auch Mitbegründer des „Verbands deutscher Kriegssammlungen" und verfasste mehrere Aufsätze zu Fragen der "Kriegssammlungen". 3

In einem Verzeichnis der Kriegssammlungen in Deutschland, das Oberstleutnant Albert Buddecke 1917 veröffentlichte, wurden für die Kriegssammlung der Stuttgarter Hofbibliothek folgende Materialien genannt:4

- Kriegsdruckschriften „Alles Erreichbare aus Kriegsgebiet und Heimat"

- Bücher „namentlich aus dem feindl. Ausland“

- Zeitschriften und Zeitungen, besonders Feldund Gefangenenlagerzeitungen

(2) Staatsarchiv Ludwigsburg, E 21 Oberhofmeisteramt, Bü 426, BI. 1.

(3) Vorbereitung und Gründung des Verbandes deutscher Kriegssammlungen e.V. In: Mitteilungen: Verband deutscher Kriegssammlungen e.V., Nr. 1 (1919), S. 2-7, hier: S. 2.

(4) Buddecke, Albert: Die Kriegssammlungen: Ein Nachweis ihrer Einrichtung und ihres Bestandes. Oldenburg: Stalling, 1917, S. 49.

(5) Die Kriegssammlung der Stuttgarter Kgl. Hofbibliothek. In: Mitteilungen: Verband deutscher Kriegssammlungen e.V., Nr. 1 (1919), S. 29-31, hier: S. 30

(6) Staatsarchiv Ludwigsburg, E 21 Oberhofmeisteramt, Bü 426, BI. 2.

(7) Die Kriegssammlung der Stuttgarter Kgl. Hofbibliothek. In: Mitteilungen. Verband deutscher Kriegssammlungen e.V., Nr. 1 (1919), S. 29-31, hier: S. 30.
- Kriegsdrucksachen wie z.B. „Entlausungsscheine, Fahrpläne, Fahrkarten, Veröffentlichungen heimatlicher Behörden"

- Kriegsandenken wie „Münzen, Marken, Exlibris, Vivatbänder, Spielzeug, Autographen, Fliegerabwürfe u.a."

\section{Stuttgart. Säniglidje soibibliothet.} St rieg i f a m I u $\mathrm{n}$.

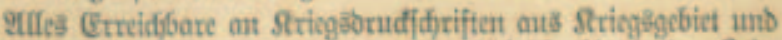

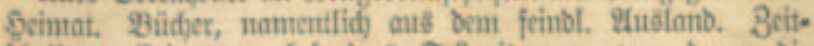

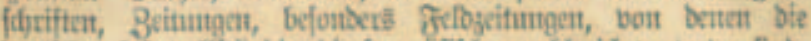

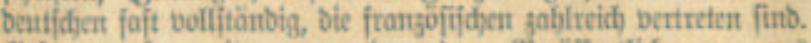
Ocfangententagerzeinumgen umb anbete Peröfientlidumgen mts

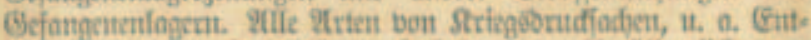

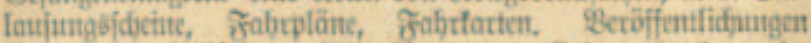

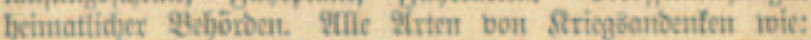

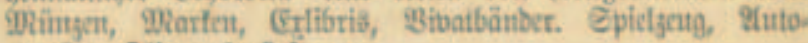
grapben, OIliegerabivitrfe u. a.

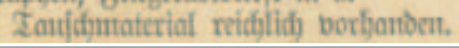

Beschreibung der Kriegssammlung der Stuttgarter Hofbibliothek, aus: Buddecke, Albert: Die Kriegssammlungen: Ein Nachweis ihrer Einrichtung und ihres Bestandes. Oldenburg: Stalling, 1917, S. 49.

Aus der Stuttgarter Sammlung wurden an anderer Stelle besonders hervorgehoben die "seltenen hektographierten" Feldzeitungen, die „vielen Tausende von Maueranschlägen, die von Ostende bis zur Krim, von Riga bis Udine in fast allen europäischen Sprachen durch deutsche Ortskommandanturen herausgegeben wurden", die „Kriegsbriefmarken in vollständigen Sätzen", die „Lebensmittelmarken der deutschen Städte, darunter die der gesamten Stuttgarter Versorgung" sowie das Notgeld. ${ }^{5} 1916$ wurde ein zweiseitiges Verzeichnis der Sammlung gedruckt und hier finden sich auch noch andere Kuriosa der Sammlung wie zum Beispiel "Schützengrabenreliquien, d. i. Arbeiten in Holz, Kreide Ton, Baumrinde". . Gemeint sind Arbeiten, die von Soldaten an der Front, von Verwundeten oder Kriegsgefangenen hergestellt wurden. Objekte dieser Art subsumiert man heute unter dem Begriff "Trench Art” ( „Schützengraben-Kunst").

Zusammenfassend hieß es an einer weiteren Stelle über die Stuttgarter Sammlung: „Stuttgart verfügt jedoch jetzt schon über so viele seltene und kostbare Stücke, daß die Berechtigung seiner Sammlung mehr als nachgewiesen erscheint" - offensichtlich keine Selbstverständlichkeit, da bei der großen Zahl an Kriegssammlungen große Überschneidungen bei den Materialien, die gesammelt wurden, nicht ausblieben. ${ }^{7}$ 
Sehr umfangreich muss auch die Fotosammlung der Stuttgarter Kriegssammlung gewesen sein. Die Stuttgarter Sammlung besitze auf diesem Gebiet "bemerkenswerte und einzigartige Schätze", die „durchweg geordnet sind“. 8 Die Fotos ließen sich drei großen Gruppen zuordnen: „die Aufnahmen des Bild- und Filmamts in Berlin, die von Berufsphotographen und Illustrationsgesellschaften eingereichten und amtlich zensierten Bilder und private Photographien. "9

Neben öffentlichen Mitteln standen der Kriegssammlung der Hofbibliothek auch Spenden von Privatleuten zum Aufbau der Sammlung zur Verfügung. So spendete beispielsweise der Pfullinger Mäzen Louis Laiblin (1861-1927) von April 1916 bis 1920 insgesamt 30.000 Mark für diese Zwecke. ${ }^{10}$ Die Kriegssammlung wurde auch von Freunden und Gönnern der Hofbibliothek unterstützt, die sich in der "Gesellschaft zur Förderung einer vaterländischen Kriegssammlung " zusammengefunden hatten. ${ }^{11}$

\section{Öffentliche Präsentationen der Kriegssammlung}

Schon im Ersten Weltkrieg wurden Teile der Kriegssammlung der Hofbibliothek Stuttgart auch öffentlich präsentiert. Vom 1. Mai bis 30. Juni 1915 fand im Landesgewerbemuseum die Ausstellung „Krieg und Kunstgewerbe" statt, zu der neben zahlreichen anderen Institutionen auch die Königliche Hofbibliothek Materialien beisteuerte. ${ }^{12}$ Gezeigt wurden Druckgraphik, graphisch besonders gestaltete Bücher, Plakate sowie Postkarten. ${ }^{13}$ Aus den Beständen der Kriegssammlung der Hofbibliothek stammten zum Beispiel die "Liller Kriegsflugblätter" u.a. mit Beiträgen des Zeichners Karl Arnold, der zahlreiche Karikaturen im "Simplicissimus" veröffentlichte. ${ }^{14}$ Welche der gezeigten Bestände daneben noch aus der Kriegssammlung der Hofbibliothek stammten, muss offen bleiben.

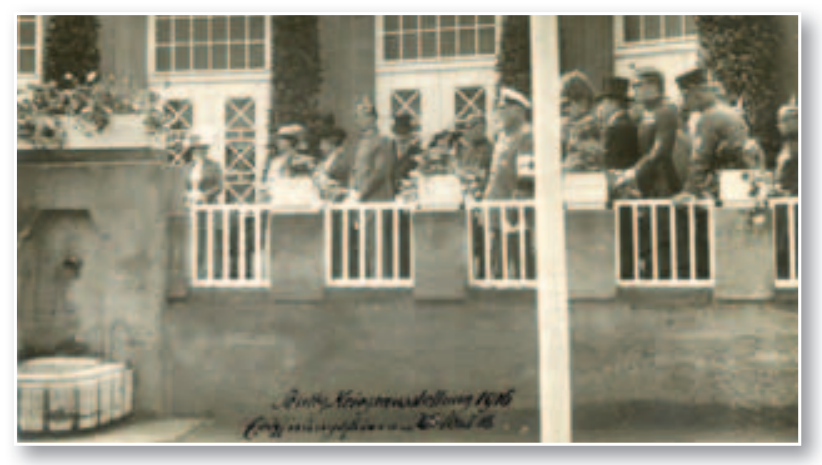

Eröffnung der Kriegsausstellung in Stuttgart, 25. Mai 1916
Die Kriegssammlung der Hofbibliothek war daneben auch auf der Deutschen Kriegsausstellung in Stuttgart vertreten. In der am 25. Mai 1916 eröffneten Ausstellung richtete die Königliche Hofbibliothek zusammen mit der Kommunalen Vereinigung die Abteilung fünf, den literarisch-graphischen Teil der Ausstellung ein. ${ }^{15}$ Aus dem Bericht über die Kriegsausstellung lassen sich auch Rückschlüsse auf die Sammlung insgesamt ziehen. So hieß es in der "Schwäbischen Kronik" über die Stuttgarter Kriegssammlung: „Es ist unseren Lesern bekannt, daß die Hofbibliothek die bedeutsame Aufgabe, die in Stuttgart nicht übersehen werden durfte, zeitig auf sich genommen hat, die literarischen Dokumente der Kriegszeit im weitesten Sinn zu sammeln. Ihr Erfolg ist erfreulicherweise immer schöner geworden, die Sammlung wächst von Tag zu Tag um wertvolle Stücke und ist heute unter den ersten im Reich. Besonders ältere, heute schon seltene Drucke weist sie in großer Reichhaltigkeit auf. Neben eigentlichen ,literarischen' Gegenständen [...] hat sie ihr Gebiet bald auch auf die Graphik, die Kriegsdenkmünzen und Plaketten ausgedehnt". ${ }^{16}$ Aus den Gesamtbeständen hatte die Bibliothek eine Auswahl getroffen, die auf der Kriegsausstellung präsentiert wurde. Einige besondere Stücke wurden in dem Zeitungsbericht näher beschrieben, so zum Beispiel „eine sehr reiche Sammlung der Maueranschläge aus der Insterburger Russenzeit", einzelne Zeitungsblätter, Witzblätter, Feldzeitungen, graphische Blätter wie zum Beispiel das amtliche württembergische Gedenkblatt von Robert Haug -, Schattenrisse von Carlos Tips, Zeichnungen, Radierungen und Ähnliches, z.B. von Slevogt, Liebermann oder auch Käthe Kollwitz. In der Sammlung von Gedenkmünzen bzw. -medaillen wurden vor allen Dingen Medaillen von Ludwig Gies und Medaillen der Stuttgarter Metallwarenfabrik Wilhelm Mayer und Franz Wilhelm gezeigt.

(8) Sperling, Eva: Aus der Sammlung von Kriegslichtbildern der Stuttgarter Hofbibliothek. In: Mitteilungen: Verband deutscher Kriegssammlungen e.V., Nr. 1 (1920), S. 5-6, hier: S. 5.

(9) Sperling, Eva: Aus der Sammlung von Kriegslichtbildern der Stuttgarter Hofbibliothek. In: Mitteilungen: Verband deutscher Kriegssammlungen e.V., Nr. 1 (1920), S. 5-6, hier: S. 5.

(10) Taigel, Hermann: Louis Laiblin: Privatier. Ein schwäbischer Mäzen. Pfullingen: Taigel, 2004 (Beiträge zur Pfullinger Geschichte; 14), S. 127.

(11) Staatsarchiv Ludwigsburg, E 21, Bü 426, BI. 21.

(12) Kgl. Landesgewerbe-Museum: Bericht über die Jahre 1914 und 1915. Stuttgart: Grüninger, [1916], S. $60 f$.

(13) Rapp, H.: Die Graphik auf der Ausstellung "Krieg und Kunstgewerbe" im Landesgewerbemuseum in Stuttgart. In: Das Plakat, Jg. 6 (1915), S. $168 f$.

(14) Krieg und Kunstgewerbe. In: Gewerbeblatt aus Württemberg, Jg. 67 (Nr. 25, 19.6.1915), S. 173-176.

(15) Schwäbische Kronik, Nr. 216 (10.5.1916), Abendblatt, S. 1

(16) Schwäbische Kronik, Nr. 282 (20.6.1916), Morgenblatt, S. 2. 
Eine andere Ausstellung beschäftigte sich nur mit dem Thema Notgeld. Sie wurde vom Landesgewerbemuseum Stuttgart vom 24. Mai bis 31. Juli 1918 gezeigt. Die Beispiele stammten alle aus der Kriegssammlung der Hofbibliothek. ${ }^{17}$ Gezeigt wurden sowohl Papierscheine als auch Hartgeld aus dem In- und Ausland. ${ }^{18}$ Abbildungen von Notgeld aus der Stuttgarter Sammlung finden sich in einem Beitrag zum Thema "Notgeld“ von Gustav E. Pazaurek, einem Mitarbeiter des Landesgewerbemuseums. ${ }^{19}$

Ebenfalls im Landesgewerbemuseum wurde vom 7. Oktober bis 6. November 1918 die Ausstellung „Bildpostkarten unserer Gegner und Kriegsmedaillen" gezeigt, die aus der Kriegssammlung der Hofbibliothek stammten. ${ }^{20}$ Das ,Stuttgarter Neue Tagblatt' brachte einen ausführlichen Bericht über diese Ausstellung und hob dabei besonders italienische Karikaturen hervor: „Sie sind von einer geradezu empörenden Ekelhaftigkeit und einer durch und durch verfaulten sexuell-perversen Phantasie. Man sollte es nicht für möglich halten, daß Menschen- oder gar (Künstler-)Sein auf solch raffiniert gemeine Machwerke kommen kann. “21 Solche und ähnliche Karikaturen hatte auch der Schriftsteller Anton Fendrich aus Freiburg in der Kriegssammlung gesehen. Seiner Meinung nach sollte man diese Karikaturen durch eine Ausstellung einem breiten Publikum bekannt machen, um den Hass auf die Feinde weiter zu schüren:

(17) Landes-Gewerbe-Museum: Bericht über die Jahre 1916-1921. Stuttgart: Grüninger, [1921], S. 41.

(18) Schwäbische Kronik, Nr. 241 (25.5.1918), S. 2

(19) Pazurek, Gustav E.: Notgeld. In: Zeitschrift des Deutschen Vereins für Buchwesen und Schrifttum, Jg. 1, Nr. 9/10 (September / Oktober 1918), S. 106-112, Hinweis auf die Kriegsbibliothek der Hofsammlung: S. 108.

Da die abgebildeten Scheine anhand der Seriennummern nicht mit den Scheinen identisch sind, die sich heute in der Notgeldsammlung der Bibliothek für Zeitgeschichte befinden, ist relativ sicher, dass es sich bei dieser Notgeldsammlung nicht um die Notgeldsammlung der Königlichen Hofbibliothek handeln kann.

(20) Landes-Gewerbe-Museum: Bericht über die Jahre 1916-1921. Stuttgart: Grüninger, [1921], S. 41.

(21) Stuttgarter Neues Tagblatt, Nr. 531 (19.10.1918), Morgenausgabe, 2. Blatt

(22) Staatsarchiv Ludwigsburg, E 21 Oberhofmeisteramt, Bü 426, BI. 2.

(23) Maier, Walter: Die Hofbibliothek Stuttgart nach dem Ende der Monarchie in Württemberg 1919-1944. Stuttgart: Württembergische Landesbibliothek, 1987, S. 5.

(24) Maier, Walter: Die Hofbibliothek Stuttgart nach dem Ende der Monarchie in Württemberg 1919-1944. Stuttgart: Württembergische Landesbibliothek, 1987, S. 12.

(25) Antiquitäten-Zeitung: Zentral-Organ für Samme/wesen, Versteigerungen und Altertumskunde, Jg. 27, Nr. 16 (1919), S. 132.

(26) Verhandlungen des Württembergischen Landtags, 44. Sitzung, Stuttgart, Dienstag, den 22. Juli 1919. In: Verhandlungen der verfassunggebenden Landesversammlung beziehungsweise des Landtags des freien Volksstaates Württemberg in den Jahren 1919 und 1920, Protokoll-Band 2, Stuttgart: Grüninger, 1920, S. 975-1006, hier: S. 1001-1003.
„Eine Ausstellung dieser Karikaturen [...] wäre eine so vornehme Rache und Antwort und eine so wirkungsvolle Belehrung aller Nur-Aestheten und Friedenshetzer in Deutschland [...]".22

\section{Die Kriegssammlung der Hofbibliothek nach 1918}

Nach der Revolution von 1918 wurde die Überleitung der Hofbibliothek schon am 29. November in einem Abkommen zwischen dem König und der Provisorischen Regierung geregelt. ${ }^{23}$ Mit insgesamt ca. 110.000 Bänden war die Bibliothek auch weiterhin in den Räumen der früheren Hohen Karlsschule untergebracht. In einem Bericht der Landesbibliothek, die die vollständige Übernahme der nun ehemaligen Hofbibliothek ablehnte, wurde als eine zukünftige Hauptaufgabe der Hofbibliothek der Ausbau der Kriegssammlung genannt. ${ }^{24}$ 1919 erklärte sich die Stadt Stuttgart bereit, die ehemalige Hofbibliothek zu übernehmen, jedoch war der Landtag der Meinung, die Sammlung solle der Landesbibliothek als besondere Abteilung angegliedert werden. ${ }^{25}$ In der Landtagsdebatte am 22. Juli 1919 kam die Rede mehrmals auch auf die von Stockmayer angelegte Kriegssammlung. Der Zentrums-Abgeordnete Dr. Baur meinte, es sei „eine ganz ausgezeichnete Kriegsbibliothek" entstanden, während sein Parteifreund, der Abgeordnete Hanser, Stockmayers Sammlung als „wohl die beste und reichhaltigste Kriegssammlung von ganz Deutschland“ bezeichnete: „Er hat gesammelt Broschüren, Zeitungen, Zeitschriften, gegen 30000 Plakate, Aufrufe, Kriegsgeld, Briefmarken, Denkmünzen, kurz alles, was in Zusammenhang mit dem Kriege steht, und was jedenfalls für spätere Geschichtsschreiber von großem Interesse ist. Und alle diese Sammlungen hat er aus Beiträgen von Gönnern, die er selber zu Stiftungen und Spenden veranlaßt hat, zusammengebracht. Die Sammlung, die er mit einer Ausgabe von etwa $30000 \mathrm{M}$ gesammelt hat, stellt heute mindestens einen Wert von einer Million Mark dar. Es sind Stücke in dieser Sammlung, die zu Beginn des Krieges zu 30 M gekauft worden sind und heute schon den zehnfachen Wert für Sammler haben. "26 Die Sammlung solle auch weiterhin fortgesetzt und gepflegt werden.

Bis auf Weiteres bestand die Hofbibliothek also fort, sie wurde jedoch an die Landesbibliothek angelehnt, indem ihr Haushalt in den Etat der Lan- 
desbibliothek aufgenommen wurde. Auch wurden bei Ordnungsarbeiten einige wertvolle kleinere Bestände an die Landesbibliothek abgegeben. ${ }^{27}$ In einem Schreiben aus dem Jahr 1922 bestand Stockmayer strikt darauf, dass „die ganze Kriegssammlung in allen ihren Teilen so bestehen bleibe", wie er sie angelegt habe. ${ }^{28}$ Die Ausgestaltung der Kriegssammlung der Hofbibliothek sei sein "Lebenswerk", meinte Stockmayer in einem Brief vom November 1918. ${ }^{29}$ Nach dem Tod des letzten württembergischen Königs Wilhelms II. (1921) entwickelte sich ein Streit zwischen den Privaterben des Königs und dem württembergischen Staat um die Kriegssammlung. Die Erben beanspruchten die Kriegssammlung als Privateigentum des Königs für sich. Der Streit konnte durch einen Vertrag 1924 beigelegt werden: Die Privaterben durften einige Stücke, die dem König geschenkt worden waren, auswählen. ${ }^{30} 1924$ wurde die Hofbibliothek rechtlich der Landesbibliothek unterstellt. Als Stockmayer Ende Juni 1936 in den Ruhestand trat, ging die Verwaltung der Hofbibliothek auf die Württembergische Landesbibliothek über. ${ }^{31}$ Die Hofbibliothek blieb jedoch nach wie vor im Gebäude der Akademie untergebracht, bis 1937 rund 10.500 Bände aus den Erscheinungsjahren 1600 bis 1750 aus Sicherheitsgründen in die Landesbibliothek verbracht wurden. Die verbliebenen Bände zogen nach Kriegsbeginn im September 1939 in das Hauptgebäude um.

Am 1. April 1936 wurde zwischen dem württembergischen Staat und der Weltkriegsbücherei, die seit 1921 im Schloss Rosenstein in Stuttgart untergebracht war, die Übernahme der Kriegssammlung der Hofbibliothek durch die Weltkriegsbücherei als Dauerleingabe vertraglich geregelt. ${ }^{32}$ Professor von Stockmayer hatte sich vehement gegen diese Übernahme ausgesprochen: Zum einen werde staatliches Eigentum einer privaten Einrichtung übergeben, zum anderen befürchtete er die Zerschlagung der Sammlung. ${ }^{33}$ Er selbst plädierte für die geschlossene Übergabe der Sammlung an das Heeresarchiv. Teile der Fotosammlung und Exemplare von beschlagnahmten Büchern wurden 1938 tatsächlich an das Heeresarchiv abgegeben. ${ }^{34}$ Archivalien aus dem Bereich des Heeres sollten nicht an eine private Einrichtung abgegeben werden, so argumentierte das Heeresarchiv. ${ }^{35}$ Immerhin wurde Stockmayer dann in die Übergabe an die Weltkriegsbücherei mit eingebunden: Er sollte nach seiner Pensionierung den Abgleich der Bestände sowie die Katalogisierung vornehmen und die Überführung und Unterbringung der Bestände in den Räumen der Weltkriegsbücherei beaufsichtigen. ${ }^{36}$ Die Bestände der Kriegssammlung der Hofbibliothek wurden Ende 1938 in das Gebäude der Weltkriegsbücherei verlagert, die Rede ist von „etwa 10000 Plakaten, Flug- und Werbeblättern, Karten, Notgeldsammlung, Gefangenenarbeiten, Briefmarkensammlung, Werbemarken, Postkarten, Aufrufen, amtlichen Drucksachen usw." 37 Auch diese Bestände wurden nach Angaben von Walter Maier sämtlich ein Opfer des Bombenangriffes auf das Schloss Rosenstein vom September 1944. ${ }^{38}$

\section{Reste der Kriegssammlung im heutigen Bestand der Bibliothek für Zeitgeschichte}

Teile der Kriegssammlung der Hofbibliothek sind jedoch offensichtlich bei der Zerstörung der Weltkriegsbücherei gerettet worden. In den heutigen Beständen der Bibliothek für Zeitgeschichte lassen sich einige kleine Reste der damaligen Kriegssammlung der Stuttgarter Hofbibliothek wiederfinden. So stammt die Sammlung der Postwertzeichen zum allergrößten Teil aus der Kriegssammlung der Hofbibliothek. Die Sammlung wurde durchaus auch noch über die Zeit nach 1918 hinaus fortgesetzt. Die jeweiligen Postwertzeichen sind auf starke Kartonbögen geklebt. Die einzelnen Blätter sind mit einer Überschrift versehen sowie mit einer Marke verziert, die die Aufschrift "Kgl. Württ. Hofbibliothek. Kriegssammlung" trägt.

(27) Maier, Walter: Die Hofbibliothek Stuttgart nach dem Ende der Monarchie in Württemberg 1919-1944. Stuttgart: Württembergische Landesbibliothek, 1987, S. 13.

(28) Schreiben Stockmayers an C. Uhlig, Geographisches Institut der Uni Tübingen vom 28.6.1922, Universitätsarchiv Tübingen 167/52, Bl. 298-301, zit. nach: Kaiser, Alexandra: „... das Material zu sammeln, das dieser Krieg in solcher Fülle schuf wie keiner vorher." Kriegssammlungen und Kriegssammler im Ersten Weltkrieg. In: Korff, Gottfried (Hrsg.): Kasten 117: Aby Warburg und der Aberglaube im Ersten Weltkrieg. Tübingen: Tübinger Vereinigung für Volkskunde e.V., 2007 (Untersuchungen des Ludwig-Uhland-Instituts der Universität Tübingen ; 105), S. 87-115, hier: S. 102.

(29) Staatsarchiv Ludwigsburg, E 21 Oberhofmeisteramt, Bü 426, BI. 21.

(30) Maier, Walter: Die Hofbibliothek Stuttgart nach dem Ende der Monarchie in Württemberg 1919-1944. Stuttgart: Württembergische Landesbibliothek, 1987, S. 42.

(31) Maier, Walter: Die Hofbibliothek Stuttgart nach dem Ende der Monarchie in Württemberg 1919-1944. Stuttgart: Württembergische Landesbibliothek, 1987, S. 29.

(32) Abschrift in: Hauptstaatsarchiv Stuttgart, M 400/1, Bü 469, BI. 14.

(33) Hauptstaatsarchiv Stuttgart, M 400/1, Bü 469, BI. 8.

(34) Hauptstaatsarchiv Stuttgart, M 400/1, Bü 469, BI. 24.

(35) Hauptstaatsarchiv Stuttgart, M 400/1, Bü 469, BI. 16.

(36) Abschrift in: Hauptstaatsarchiv Stuttgart, M 400/1, Bü 469, BI. 14.

(37) Schreiben vom 23.12.1938, Archiv der BfZ, I Da 2

(38) Maier, Walter: Die Hofbibliothek Stuttgart nach dem Ende der Monarchie in Württemberg 1919-1944. Stuttgart: Württembergische Landesbibliothek, 1987, S. 46. 


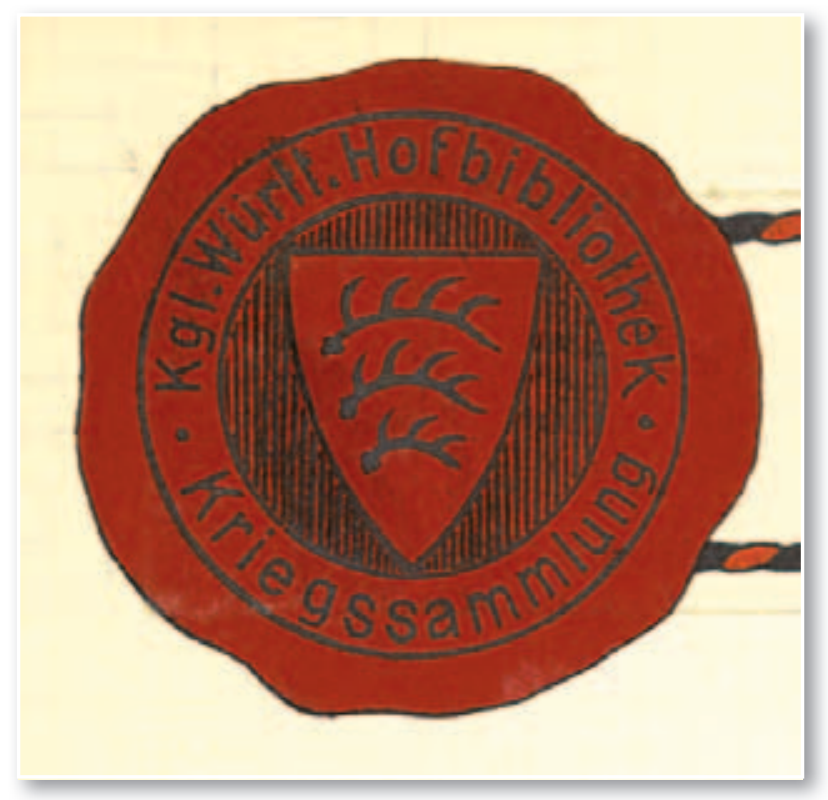

Siegelmarke der Kriegssammlung der Hofbibliothek

Die Überschriften der einzelnen Blätter beinhalten teilweise auch wertende Begrifflichkeiten der Zeit. So heißt es zum Beispiel über Postwertzeichen zur Volksabstimmung in Nordschleswig 1920: „Erinnerungsmarken zur Einverleibung von Nordschleswig". Insgesamt umfasst die Sammlung 473 Blätter mit ca. 1.330 aufgeklebten Postwertzeichen aus 36 verschiedenen Ländern. Am häufigsten sind in der Sammlung naturgemäß deutsche Postwertzeichen vertreten - insgesamt 375 Marken. An zweiter Stelle steht erstaunlicherweise die Türkei mit 179 Marken. Die Türkei war im Ersten Weltkrieg Verbündeter des Deutschen Reichs und Österreich-Ungarns. Aus Österreich-Ungarn gibt es in der Sammlung ebenfalls zahlreiche Marken, aber auch exotischere Länder wie Litauen, Malta oder Gibraltar sind vertreten. Neben einzelnen Postwertzeichen wurden teilweise auch sog. Ganz-sachen gesammelt, andere Rubriken enthalten "Zensurstempel” und „Kuriosa" wie zum Beispiel eine "Weihnachtssendung vom Schwäbischen Frauenverein in San Franzisko an Ihre Majestät die Königin“ oder eine "deutsche Feldpostkarte mit russischer Briefmarke".

Auch der größte Teil der Sammlung der Gelegenheitsmarken der Bibliothek für Zeitgeschichte stammt aus der Kriegssammlung der Hofbibliothek. Wie die Briefmarken sind auch die Gelegenheitsmarken auf Kartonblätter aufgeklebt, auch diese tragen die Marke der Kriegssammlung der Hofbibliothek und sind mit Überschriften versehen. Insgesamt sind 575 Blatt mit 3.245 Marken aus 13 verschiedenen Ländern vorhanden. Den größten Anteil bilden Marken aus Österreich (1.040), gefolgt von Marken aus dem Deutschen Reich (750), Frankreich (653) und England (266). Bei den deutschen Marken fallen unter der Rubrik "Agitationsmarken" die zahlreichen Marken mit dem Spruch "Gott strafe England" auf.

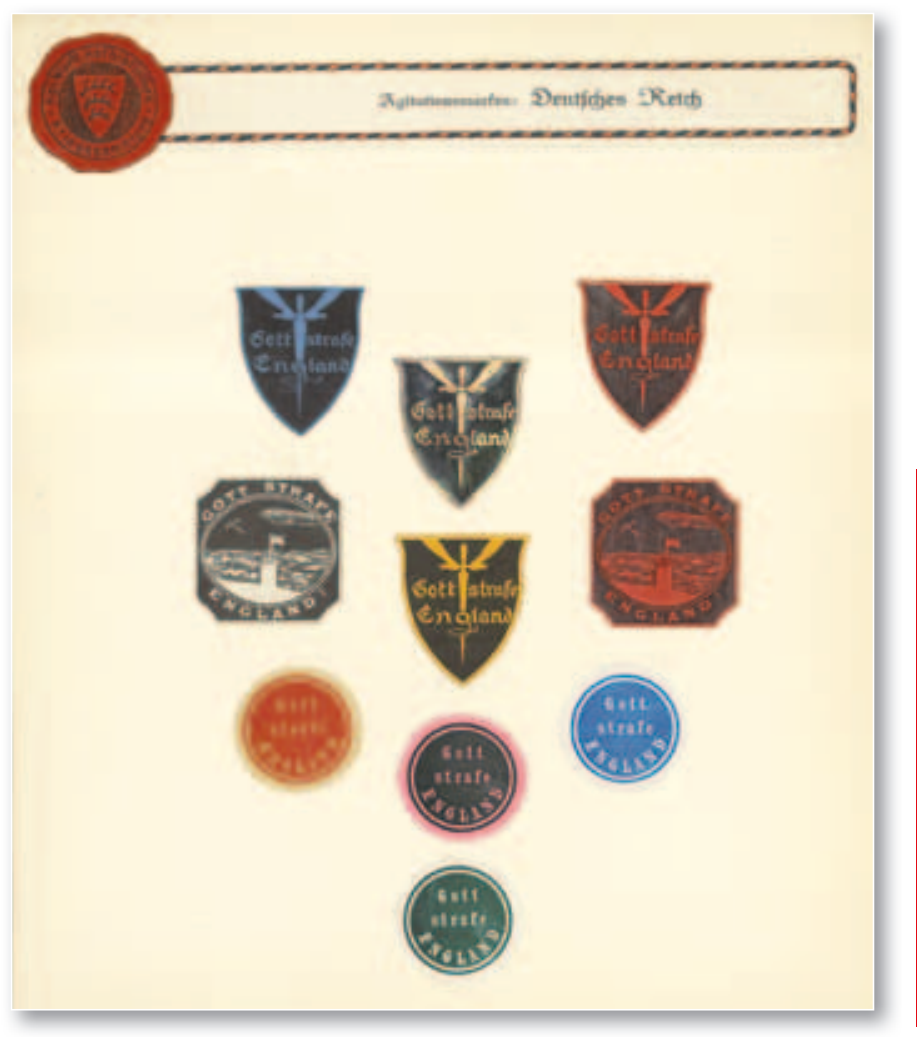

Blatt mit Werbemarken "Gott strafe England" aus der Sammlung der Bibliothek für Zeitgeschichte

Dabei handelt es sich um einen in Deutschland weit verbreiteten Spruch, der wohl aus Ernst Lissauers Gedicht "Hassgesang gegen England” entstanden war. Er diente zeitweilig auch als neue Grußformel, auf die mit "Er strafe es" geantwortet werden sollte. Die meisten der Marken insgesamt waren Wohltätigkeitsmarken, das heißt mit ihrem Kauf spendete man gleichzeitig für wohltätige Zwecke. So gibt es unter den französischen Marken beispielsweise alleine 448 „Verschlussmarken zu Gunsten französischer Regimenter".

Neben den Sammlungen von Postwertzeichen und Gelegenheitsmarken sind als weitere Stücke aus der Kriegssammlung der Stuttgarter Hofbibliothek die sogenannten „Königsalben“ zu nennen. Es handelt sich dabei um eine Sammlung privater Fotografien württembergischer Soldaten: „Das württ. Kriegsministerium hatte sich im Frühjahr 
1918 zur Herstellung eines besonderen Albums, das dem König überreicht werden sollte, am [sic!] sämtliche württembergische Truppenteile um Überlassung besonders charakteristischer Photographien aus den Kämpfen und dem Leben der Truppen gewandt. Der Aufforderung wurde im reichsten Maße entsprochen, so daß etwa 9000 Bilder für den gedachten Zweck zur Verfügung standen. Eine besondere Auswahl daraus wurde zu einem dreibändigen Album: ,Die Württemberger im Weltkrieg' vereinigt, von dem nur 1 Exemplar hergestellt und dem König überreicht wurde. Dieses sog. ,Königsalbum' kam als Geschenk des Königs an die Hofbibliothek. "39 Alle drei „Königsalben" sind heute noch vorhanden, sie liegen in der "Sammlung Zeit der Weltkriege" der Bibliothek für Zeitgeschichte (Signatur: HBb 1922-1-3). Zu diesen Alben gehören noch drei Text-Bände mit Erläuterungen sowie zwei Karten-Bände. Diese Bände stehen im Magazin der Württembergischen Landesbibliothek (Signatur AHa 164). Der erste dieser Bände, die sämtlich mit Schreibmaschine geschrieben und dann aufwändig gebunden wurden, behandelt das Jahr 1914. Dargestellt werden sollte der Anteil der württembergischen Soldaten am Kriegsgeschehen, wobei ihre Taten schon im Vorwort des ersten Bandes glorifiziert wurden: „überall, wo unsere Württemberger kämpften, bedeckten sie sich mit unsterblichem Ruhm, ihre herrlichen Taten müssen uns mit Bewunderung erfüllen, sie suchen ihresgleichen". ${ }^{40}$ Sämtliche Königsbände "Die Württemberger im Weltkrieg” sowohl Text- als auch Bildbände - sind Unikate. Die weiteren 8.000 Fotos, die bei der Sammlung zusammenkamen und die nicht in die „Königsalben" aufgenommen wurden, zog man auf Kartons auf und vereinigte diese in ca. 25 thematisch geordnete Sammelalben. So gab es beispielsweise auch ein sog. „Argonnenalbum” der 27. (2. Königlich Württembergischen) Division. ${ }^{41}$ Diese und andere Alben kamen ebenfalls in den Besitz der Kriegssammlung. Offensichtlich gehörten diese Bilder zu den Beständen, die 1938 an das Heeresarchiv Stuttgart gingen, sie liegen heute im Hauptstaatsarchiv Stuttgart. ${ }^{42}$

Aus der riesigen Kriegssammlung der Stuttgarter Hofbibliothek hat nur ein sehr kleiner Teil überlebt. Aber nicht der uninteressanteste: Trotz der großen Vielfalt und ihrer weiten Verbreitung sind besonders die verschiedenen Marken von der Forschung bisher noch kaum untersucht worden. Die Restbestände aus der Kriegssammlung der Stuttgarter Hofbibliothek bieten dafür eine gute Quellenbasis.

Hans-Christian Pust
(39) Sperling, Eva: Aus der Sammlung von Kriegslichtbildern der Stuttgarter Hofbibliothek. In: Mitteilungen: Verband deutscher Kriegssammlungen e.V., Nr. 1 (1920), S. 5-6, hier: S. 5.

(40) Die Württemberger im Weltkrieg [maschinenschriftliches Manuskript], Bd. 1. [Stuttgart], [1918], S. 5.

(41) Sperling, Eva: Aus der Sammlung von Kriegslichtbildern der Stuttgarter Hofbibliothek. In: Mitteilungen: Verband deutscher Kriegssammlungen e.V., Nr. 1 (1920), S. 5-6, hier: S. 6.

(42) Hauptstaatsarchiv Stuttgart, M 400/1, Bü 469, BI. 24. Die Bilder liegen unter: Hauptstaatsarchiv Stuttgart, Bestand M 705/1.

Abbildungsverzeichnis:

Abb. 1: Schwäbisches Bilderblatt, Nr. 15, 10.4.1931, S. 3.

Abb. 2: Buddecke, Albert: Die Kriegssammlungen:

ein Nachweis ihrer Einrichtung und ihres Bestandes. Oldenburg: Stalling,

1917, S. 49.

Abb. 3: Sammlung Pust

Abb. 4 und 5: Sammlung der Werbemarken,

Bibliothek für Zeitgeschichte. 